Tek Kapta Yeni Bir Yöntemle Alkollerden Potansiyel Biyolojik Aktif Azidosülfonil Bileşiklerinin Sentezi

\author{
Ufuk ATMACA ${ }^{1 *}$
}

ÖZET: Bu çalışmada, çeşitli alkoller ile klorosülfonil izosiyanat (CSI) ve sodyum azid varlığında tek kapta yeni bir yöntemle azidosülfonil bileşiklerinin sentezi gerçekleştirilmiştir. Reaksiyon verimin artırılması için kullanılan asit, baz ve çözücü parametreleri incelenmiş ve optimize reaksiyon şartlarında yüksek verimlerle azidosülfonil bileşiklerinin sentezi gerçekleştirilmiştir. Sentezleri gerçekleştirilen bileşiklerin yapı karakterizasyonları ${ }^{1} \mathrm{H}$ NMR, ${ }^{13} \mathrm{C}$ NMR, IR ve Elementel analiz sonuçları ile yapılmıştır.

Anahtar Kelimeler: Azidosülfonil, alkol, klorosülfonil izosiyanat, sodyum azit

\title{
The Novel Method and One-pot Synthesis of Potential Biological Active Azidosulfonyl Compounds from Alcohols
}

\begin{abstract}
In this study, the one-pot synthesis of azidosulphonyl compounds by a new method was carried out in the presence of chlorosulfonyl isocyanate (CSI) and sodium azide with various alcohols. The acid, base and solvent parameters used to increase the reaction yield were investigated and synthesis of azidosulfonyl compounds was carried out in high yields under optimized reaction conditions. The structure characterization of the synthesized molecules was performed with ${ }^{1} \mathrm{H} N \mathrm{NMR},{ }^{13} \mathrm{C} \mathrm{NMR}$, IR and Elemental analysis.
\end{abstract}

Keywords: Azidosulphonyl, alcohol, chlorosulfonyl isocyanate, sodium azide

${ }^{1}$ Ufuk ATMACA (Orcid ID: 0000-0002-5598-0443), Atatürk Üniversitesi, Oltu Meslek Yüksek Okulu, Erzurum-Oltu, Türkiye

*Sorumlu Yazar/Corresponding Author: Ufuk ATMACA, e-mail: uatmaca@atauni.edu.tr 


\section{GíRiş}

Azidosülfonil, sülfon grubuna bir amino ve bir azit grubunun bağlı olduğu biyolojik aktiviteye sahip bileşiklerdir (Hartwig 1998). Bağlı oldukları bu gruplar ilaç olma özelliği kazandırmakta ve günümüzde anti-kanser tedavisinde, antibakteriyel, antiflemetuar, antiviral vb. kullanılan ilaçların yapılarında bulunduğu bilinmektedir (Goddard-Borger ve Stick 2007; Matier ve ark., 1972). Bu sebeple azidosülfonil bileşikleri hem mekanistik organik kimya alanında hem de kullanım alanları bakımından sentetik farmasötik kimya alanında oldukça önemli bir yere sahiptirler (Daryadel ve ark., 2018). Literatürde sentezleri gerçekleştirilen azidosülfonil bileşiklerinin biyolojik aktivite çalışmaları (Kienle ve ark., 2007) ve aminoacyl-tRNA synthetases (aaRSs)'ın sülfamat inhibisyonu çalışmalarında önemli bulgular elde edilmiştir (Wolf ve Kirschbaum 1999; Golob ve ark., 2002).

Ayrıca azidosülfonil bileşiğinin termolizi sonucu sulfamid bileşiklerinin sentezlendiği bilinmekte ve birçok sentez yöntemi bulunmaktadır (Akbaba ve ark., 2014; Bauer ve ark., 2016; Zhao ve ark. 2016). Azidosülfamoil bileşiğindeki sülfon ve azot grupları bu bileşiklerin türevlendirilmesinde önemli fonksiyonel gruplardır (Culhane ve Fokin 2011).

\section{MATERYAL VE YÖNTEM}

\section{Materyaller ve Cihazlar}

Çalışmada kullanılan tüm çözücü ve kimyasallar ticari olarak satın alınmış ve saflaştırma yapılmadan kullanılmıştır. ${ }^{1} \mathrm{H}$ NMR (400 MHz) ve ${ }^{13} \mathrm{C}$ NMR (100 MHz) spektrumları Bruker Avance II spektrometresi ile alınmıştır. IR spektrumları Perkin-Elmer spektroforometresi ile alınmıştır. Elementel analiz sonuçları ise LECO CHNS-932 cihazı ile alınmıştır.

Azidosülfonil Bileşiklerinin sentezi için Genel Prosedür:

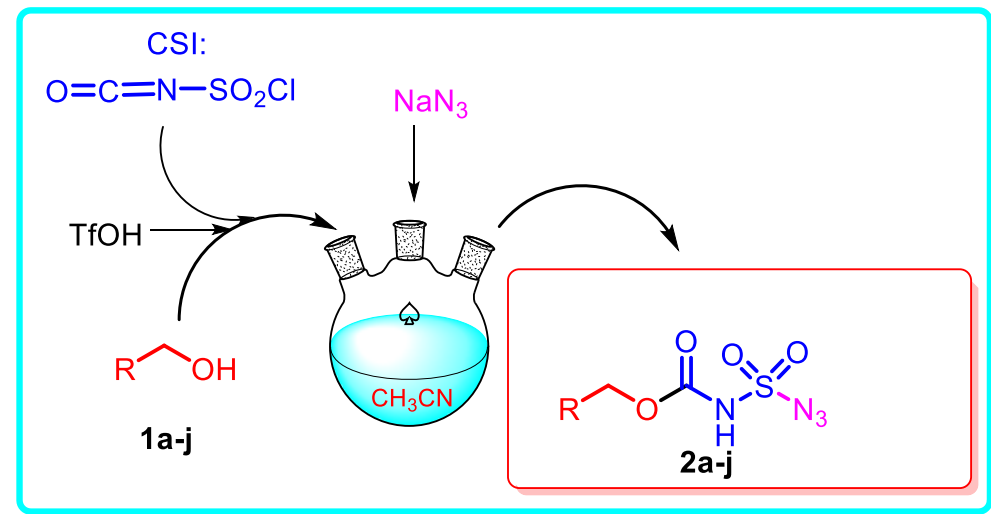

Şekil 1. Azidosülfonil bileşiklerinin sentezi için genel prosedür

İlgili alkol (1a-j) (1.0 mmol) alınarak $10 \mathrm{~mL} \mathrm{CH}_{3} \mathrm{CN}$ içinde çözüldü. Ardından reaksiyon karışımına TfOH (1.0 mmol) ilave edildi. Reaksiyon karışımı buz banyosu içerisine yerleştirildi. Reaksiyon ortamına klorosülfonil izosiyanat $(1.1 \mathrm{mmol}) 3$ dakika boyunca damla damla ilave edildi ve oda sıcaklığında reaksiyon 6 saat karıştırıldı. Ardından reaksiyon karışımı $0^{\circ} \mathrm{C}$ 'ye soğutularak üzerine sodyum azit $(1.0 \mathrm{mmol})$ ilave edildi ve reaksiyon karışımı aynı sıcaklıkta 2 saat daha karıştırıldı. Ham ürün diklorometan ile ekstraksiyonu sonucu organik çözücü sodyum sülfat ile kurutuldu ve çözücüsü vakum altında uzaklaştırıldı. Ham ürün ince tabaka kromatografisi ile EtOAc:n-hekzan (20:80) çözücüsünde ilgili azidosülfonil bileşikleri (2a-j) saflaştırıldı.

Benzil(azidosülfonil)karbamat (2a): Yoğun renksiz sıvı (\%92 verim). ${ }^{1} \mathbf{H}-\mathbf{N M R}$ (400 MHz, $\left.\mathbf{C D C l}_{3}, \mathbf{p p m}\right): \delta 5.25$ (s, 2H), 7.33-7.42 (m, 5H), 8.63 (bs, $\left.1 \mathrm{H}, \mathrm{NH}\right) .{ }^{13} \mathbf{C}-\mathbf{N M R}\left(\mathbf{1 0 0} \mathbf{~ M H z}, \mathbf{C D C l}_{3}\right.$, ppm): $\delta$ 69.7, 128.7, 128.9, 129.2, 133.9, 150.6, IR (CHCl $\left.\mathbf{3}, \mathbf{c m}^{-1}\right): 3269,2156,1753,1452,1394,1232$, 
1166, 1059. Elementel Analiz Hesaplanan: C, 37.50; H, 3.15; N, 21.87; S, 12.51 bulunan: C, 37.14; H, 3.22; N, 21.69; S, 12.35.

1-Feniletil(azidosülfonil)karbamat (2b): Yoğun renksiz sıvı (\%90 verim). ${ }^{1} \mathbf{H}-\mathbf{N M R}$ (400 MHz, Aseton-d6, ppm): $\delta 1.62(\mathrm{~d}, \mathrm{~J}=4.1,3 \mathrm{H}), 5.96(\mathrm{q}, \mathrm{J}=6.6,1 \mathrm{H}), 7.34-7.46(\mathrm{~m}, 5 \mathrm{H}) .{ }^{13} \mathrm{C}-\mathrm{NMR}(\mathbf{1 0 0} \mathbf{~ M H z}$, Aseton-d6, ppm): $\delta 22.5,76.8,126.8,129.2,129.5,141.7,151.6$. IR $\left(\mathbf{C H C l}_{\mathbf{3}}, \mathbf{c m}^{-1}\right): 3281,2151,1749$, 1450, 1382, 1169, 1060. Elementel Analiz Hesaplanan: C, 40.00; H, 3.73; N, 20.73; S, 11.86, bulunan: $\mathrm{C}, 40.15 ; \mathrm{H}, 3.89$; N, 20.47; S, 11.59.

Feniletil(azidosülfonil)karbamat (2c): Yoğun renksiz sıvı (\%88 verim). ${ }^{\mathbf{1}} \mathbf{H}-\mathrm{NMR}$ (400 MHz, CDCl3, ppm): $\delta 2.99(\mathrm{t}, \mathrm{J}=7.0,2 \mathrm{H}), 4.45(\mathrm{t}, \mathrm{J}=7.0,2 \mathrm{H}), 6.41$ (bs, $1 \mathrm{H}, \mathrm{NH}), 7.20-7.33(\mathrm{~m}, 5 \mathrm{H}) .{ }^{{ }^{13} \mathbf{C}-}$ NMR (100 MHz, CDCl3, ppm): $\delta$ 34.9, 68.2, 127.0, 128.7, 128.9, 136.6, 151.2. IR (CHCl3, $\mathbf{~ c m}^{-1}$ ): 3263, 2154, 1752, 1454, 1167. Elementel Analiz Hesaplanan: C, 40.00; H, 3.73; N, 20.73; S, 11.86, bulunan: C, 40.14; H, 3.65; N, 20.58; S, 11.93 .

Siklohekz-2-en-1-il (azidosülfonil)karbamat (2d): Yoğun renksiz sıv1 (\%83 verim). ${ }^{\mathbf{1}} \mathbf{H}-\mathbf{N M R}$ (400 MHz, CDCl3, ppm): $\delta$ 1.69-2.15 (m, 6H), 5.33 (d, J=4.8, 1H), 5.75-5.78 (m, 1H), 6.03-6.07 (m,

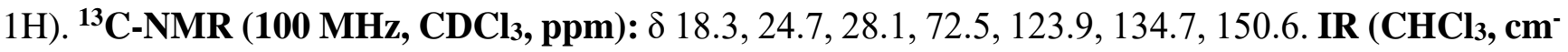
1): 3246, 2943, 2152, 1747, 1451, 1391, 1241, 1171, 1048. Elementel Analiz Hesaplanan: C, 34.14; H, 4.09; N, 22.75; S, 13.02, bulunan: C, 34.28; H, 4.12; N, 22.53; S, 13.31.

3,5,5-Trimetilsiklohek-2-en-1-il (azidosülfonil)karbamat (2e): Yoğun renksiz sıv1 (\%76 verim). ${ }^{1} \mathrm{H}-\mathrm{NMR}$ (400 MHz, CDCl3, ppm): $\delta$ 0.92-0.98 (m, 1H), 1.03 (s, 3H), 1.24 (s, 3H), 1.50 (AB sisteminin A k1smı, dd, J=2.1, 13.1, 1H), 1.95 (s, 3H), 2.05 (AB sisteminin B k1sm1, dd, J= 3.5, 13.1, 1H), 2.98 (s, 1H), 5.11-5.14 (m, 1H), 6.18-6.20 (m, 1H). ${ }^{13}$ C-NMR (100 MHz, CDCl 3 , ppm): $\delta$ 21.1, 28.8, 29.7, 33.4, 40.8, 57.9, 62.8, 123.9, 142.1, 169.3. IR (CHCl $\left.\mathbf{3}, \mathbf{~ c m}^{-1}\right): 3282,2962,2127,1748,1406$, 1194, 1139, 1056. Elementel Analiz Hesaplanan: C, 41.66; H, 5.59; N, 19.43; S, 11.12, bulunan: C, 41.52; H, 5.65; N, 19.57; S, 11.24.

Allil (azidosülfonil)karbamat (2f): Yoğun renksiz sivı (\%91 verim). ${ }^{\mathbf{1}} \mathrm{H}-\mathrm{NMR}$ (400 MHz, $\left.\mathbf{C D C l}_{3}, \mathbf{p p m}\right): \delta 4.75$ (d, J=5.96, 2H), 5.38 (m, 2H), 5.94 (ddd, J=5.96, 11.69, 16.61, 1H). 8.18 (bs, 1H). ${ }^{13}$ C-NMR (100 MHz, CDCl 3 , ppm): $\delta$ 68.5, 120.5, 130.3, 150.2. IR (CHCl3, $\left.\mathbf{~ c m}^{-1}\right): 3262,2958,2157$, 1754, 1458, 1392, 1236, 1169, 1059. Elementel Analiz Hesaplanan: C, 23.30; H, 2.93; N, 27.17; S, 15.55, bulunan: $\mathrm{C}, 23.42 ; \mathrm{H}, 2.86$; N, 27.41; S, 15.63

Büt-3-en-2-il (azidosülfonil)karbamat (2g): Yoğun renksiz sıvı (\%90 verim). ${ }^{\mathbf{1}} \mathbf{H}-\mathbf{N M R}(400$ MHz, CDCl3, ppm): $\delta 1.42$ (d, J=6.6, 3H), 5.23-27 (m, 2H), 5.30-5.44 (m, 1H), 5.53 (bs, 1H), 5.82$5.90(\mathrm{~m}, 1 \mathrm{H}) .{ }^{\mathbf{1 3}} \mathbf{C}-\mathbf{N M R}(\mathbf{1 0 0} \mathbf{~ M H z}, \mathbf{C D C l} \mathbf{3}, \mathbf{p p m}): \delta 19.8,75.9,117.9,135.8,149.9 . \mathbf{I R}\left(\mathbf{C H C l}_{3}, \mathbf{c m}^{-}\right.$ 1): 3283, 2141, 1742, 1449, 1381, 1170, 1039. Elementel Analiz Hesaplanan: C, 27.27; H, 3.66; N, 25.44; S, 14.56, bulunan: C, 27.46; H, 3.78; N, 25.32; S, 14.43 .

2-Bütil (azidosülfonil)karbamat (2h): Yoğun renksiz sıvı (\%84 verim). ${ }^{\mathbf{1}} \mathrm{H}-\mathrm{NMR}$ (400 MHz, CDCl, ppm): $\delta$ 0.95-0.99 (m, 3H), 1.33-1.50 (m, 3H), 1.66-1.74 (m, 2H), 4.93-4.98 (m, 1H). ${ }^{13} \mathbf{C}-\mathbf{N M R}$ (100 MHz, CDCl3, ppm): $\delta$ 9.5, 19.3, 28.7, 77.7, 150.3. IR (CHCl3, $\left.\mathbf{~ c m}^{-1}\right): 3253,2979,2154,2154$, 1727, 1446, 1170. Elementel Analiz Hesaplanan: C, 27.03; H, 4.54; N, 25.21; S, 14.43, bulunan: C, 27.32; H, 4.48; N, 25.13; S, 14.56

Bütil (azidosülfonil)karbamat (2i): Yoğun renksiz sıv1 (\%89 verim). ${ }^{1}$ H-NMR (400 MHz, CDCl$_{3}$, ppm): $\delta 0.95$ (t, J=7.4, 3H), 1.40 (hekzet, $\mathrm{J}=7.4,2 \mathrm{H}$ ), 1.68 (hekzet, J=6.7, 2H), 4.00 (t, J=6.7, 2H), 6.35 (bs, 1H). ${ }^{13} \mathbf{C}-\mathbf{N M R}\left(\mathbf{1 0 0} \mathbf{~ M H z}, \mathbf{C D C l}_{3}, \mathbf{p p m}\right): \delta 13.5,18.8,30.4,68.2,150.7$. IR $\left(\mathbf{C H C l}_{3}\right.$, $\left.\mathbf{c m}^{-\mathbf{1}}\right):$ 3255, 2964, 2154, 1752, 1458, 1170. Elementel Analiz Hesaplanan: C, 27.03; H, 4.54; N, 25.21; S, 14.43, bulunan: C, 27.26; H, 4.71; N, 25.12; S, 14.48 . 
Siklohekzil (azidosülfonil)karbamat (2j): Yoğun renksiz sıvı (\%86 verim). ${ }^{\mathbf{1}} \mathbf{H}-\mathbf{N M R}$ (400 MHz, CDCl3, ppm): $\delta$ 1.14-1.59 (m, 6H) 1.66-1.80 (m, 2H), 1.86-1.95 (m, 2H). 4.69-4.76 (m, 1H), 6.51

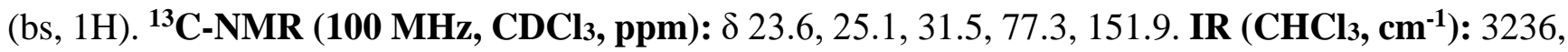
2938, 1747, 1625, 1451, 1171. Elementel Analiz Hesaplanan: C, 33.87; H, 4.87; N, 22.57; S, 12.91, bulunan: $\mathrm{C}, 33.48 ; \mathrm{H}, 4.62 ; \mathrm{N}, 22.69 ; \mathrm{S}, 13.12$

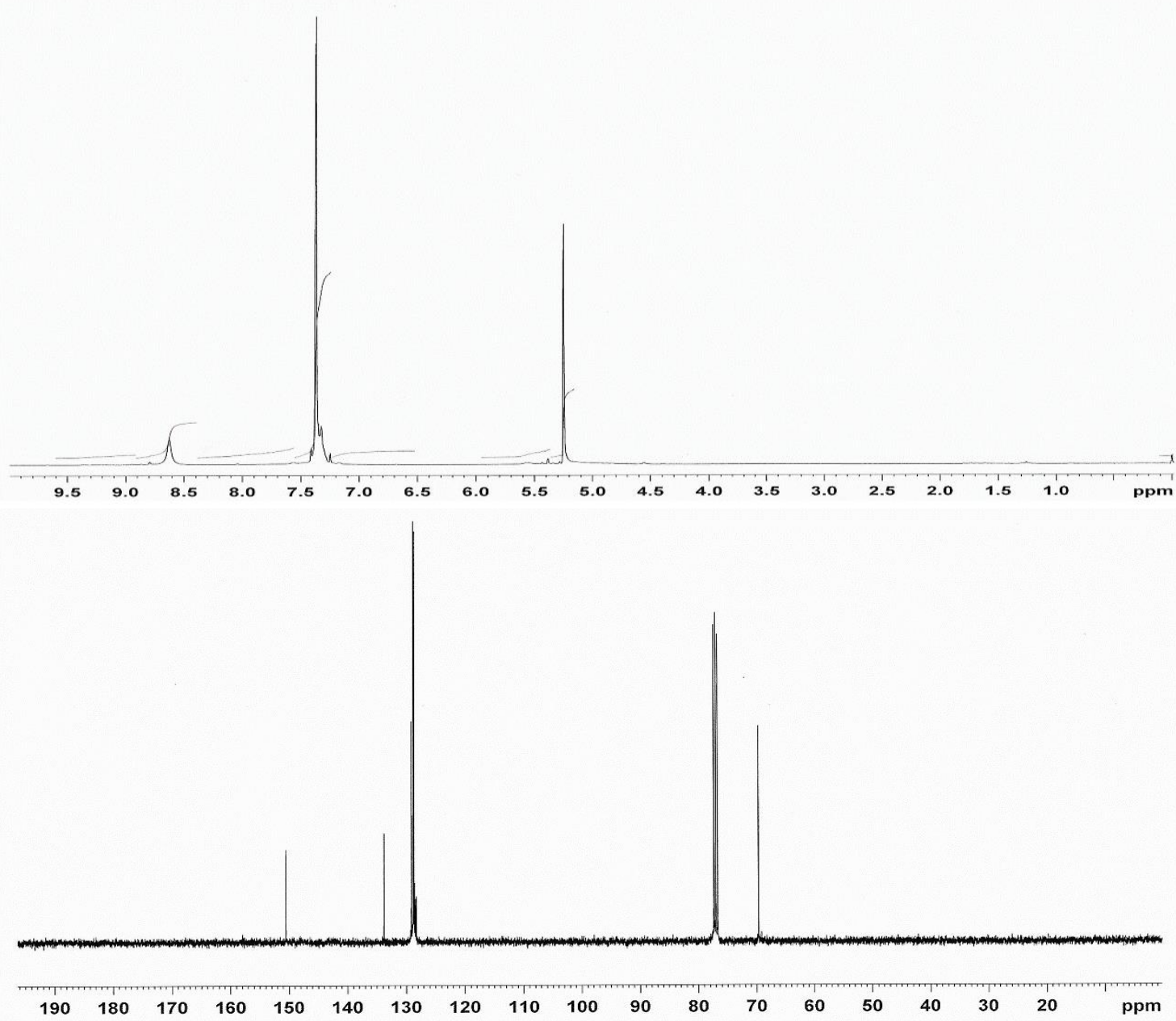

Şekil 2. Benzil(azidosülfonil)karbamat (2a) bileşiğine ait ${ }^{1} \mathrm{H}-\mathrm{NMR}$ ve ${ }^{13} \mathrm{C}-\mathrm{NMR}$ Spektrumları

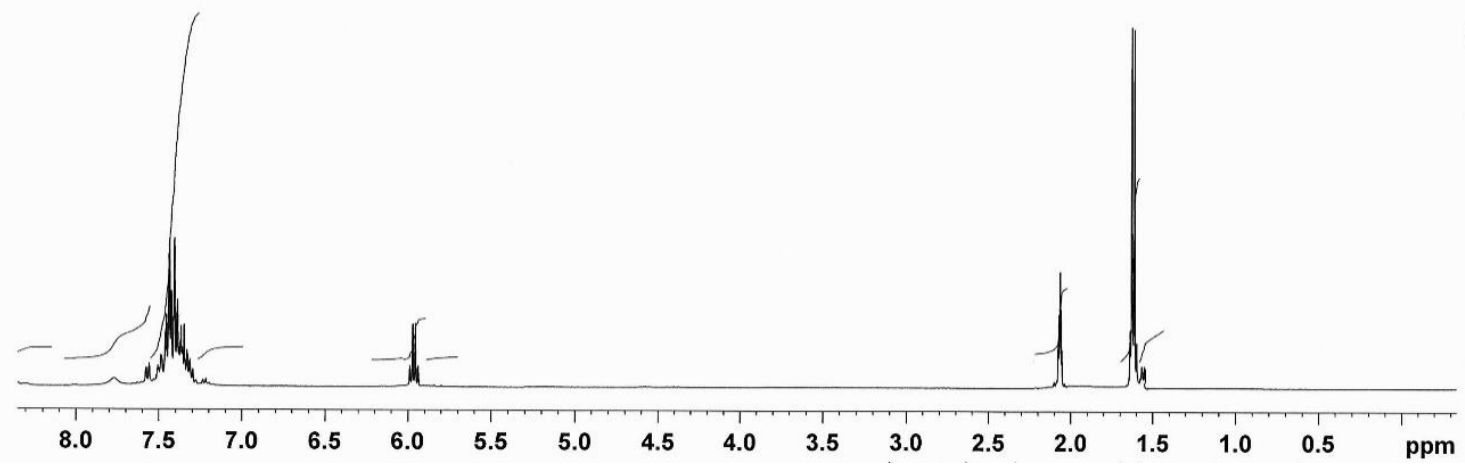

Şekil 3. 1-Feniletil(azidosülfonil)karbamat (2b) bileşiğine ait ${ }^{1} \mathrm{H}-\mathrm{NMR}$ ve ${ }^{13} \mathrm{C}-\mathrm{NMR}$ Spektrumları 


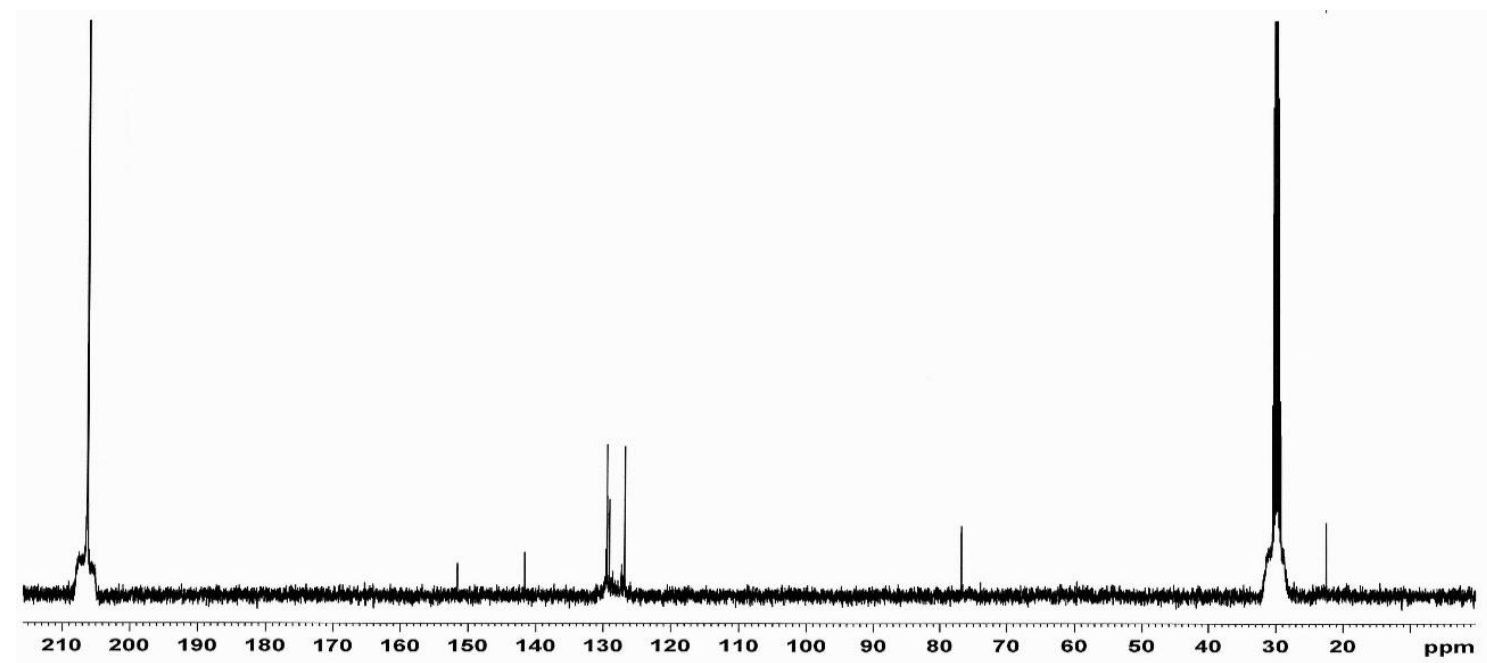

Şekil 3. 1-Feniletil(azidosülfonil)karbamat (2b) bileşiğine ait ${ }^{1} \mathrm{H}-\mathrm{NMR}$ ve ${ }^{13} \mathrm{C}-\mathrm{NMR}$ Spektrumları (devam)
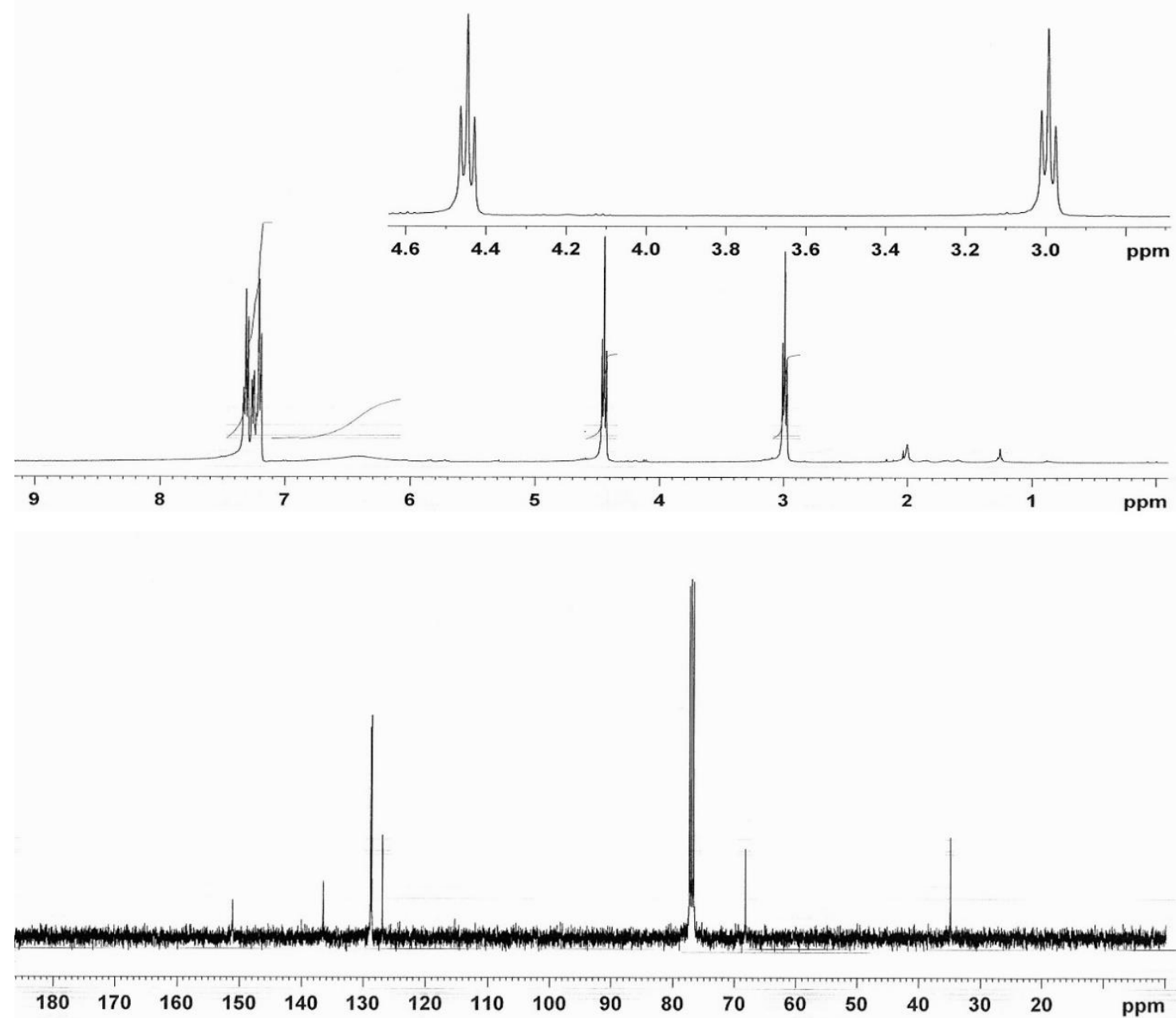

Şekil 4. Feniletil(azidosülfonil)karbamat (2c) bileşiğine ait ${ }^{1} \mathrm{H}-\mathrm{NMR}$ ve ${ }^{13} \mathrm{C}-\mathrm{NMR}$ Spektrumları 

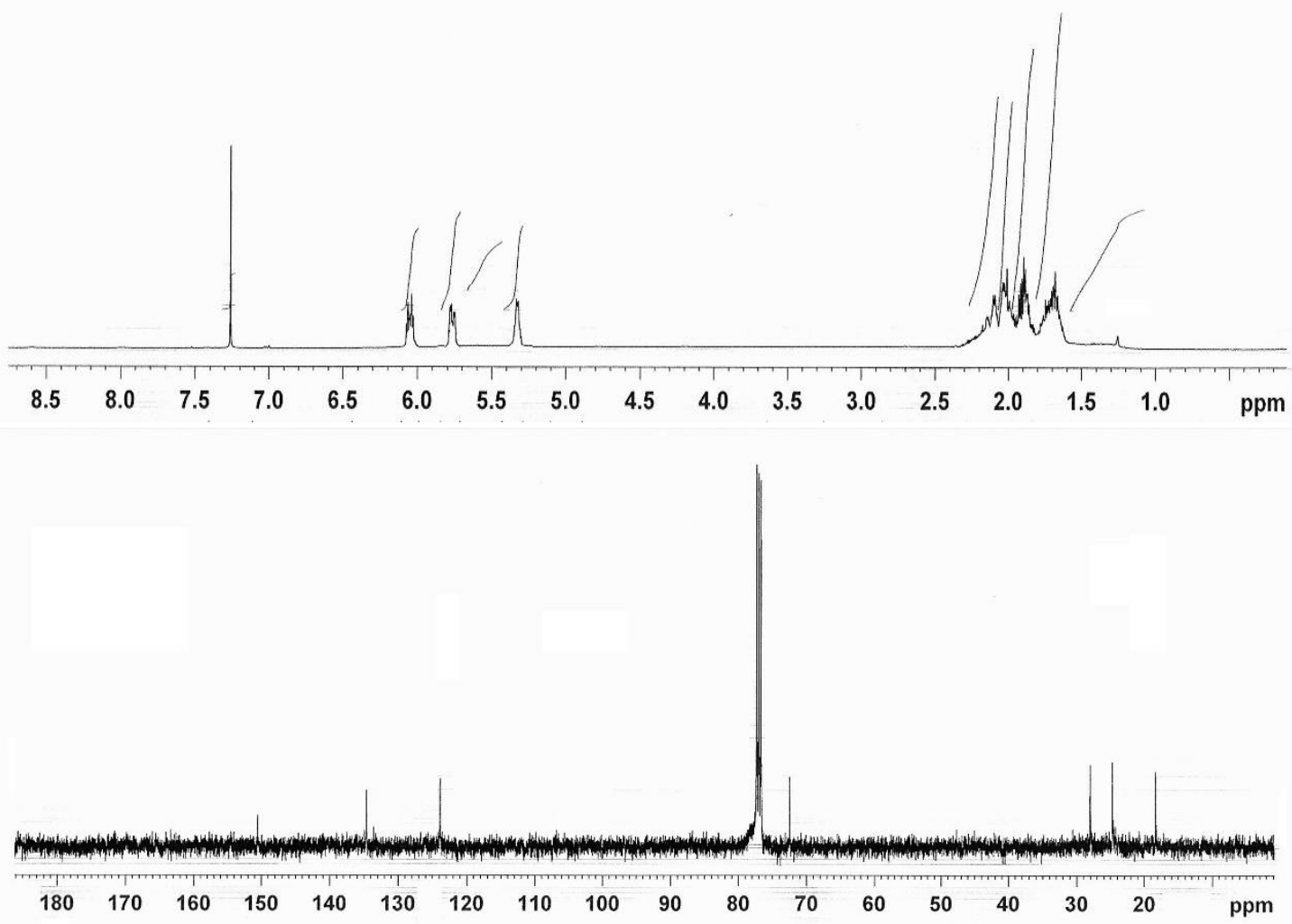

Şekil 5. Siklohekz-2-en-1-il (azidosülfonil)karbamat (2d) bileşiğine ait ${ }^{1} \mathrm{H}-\mathrm{NMR}$ ve ${ }^{13} \mathrm{C}-\mathrm{NMR}$ Spektrumları
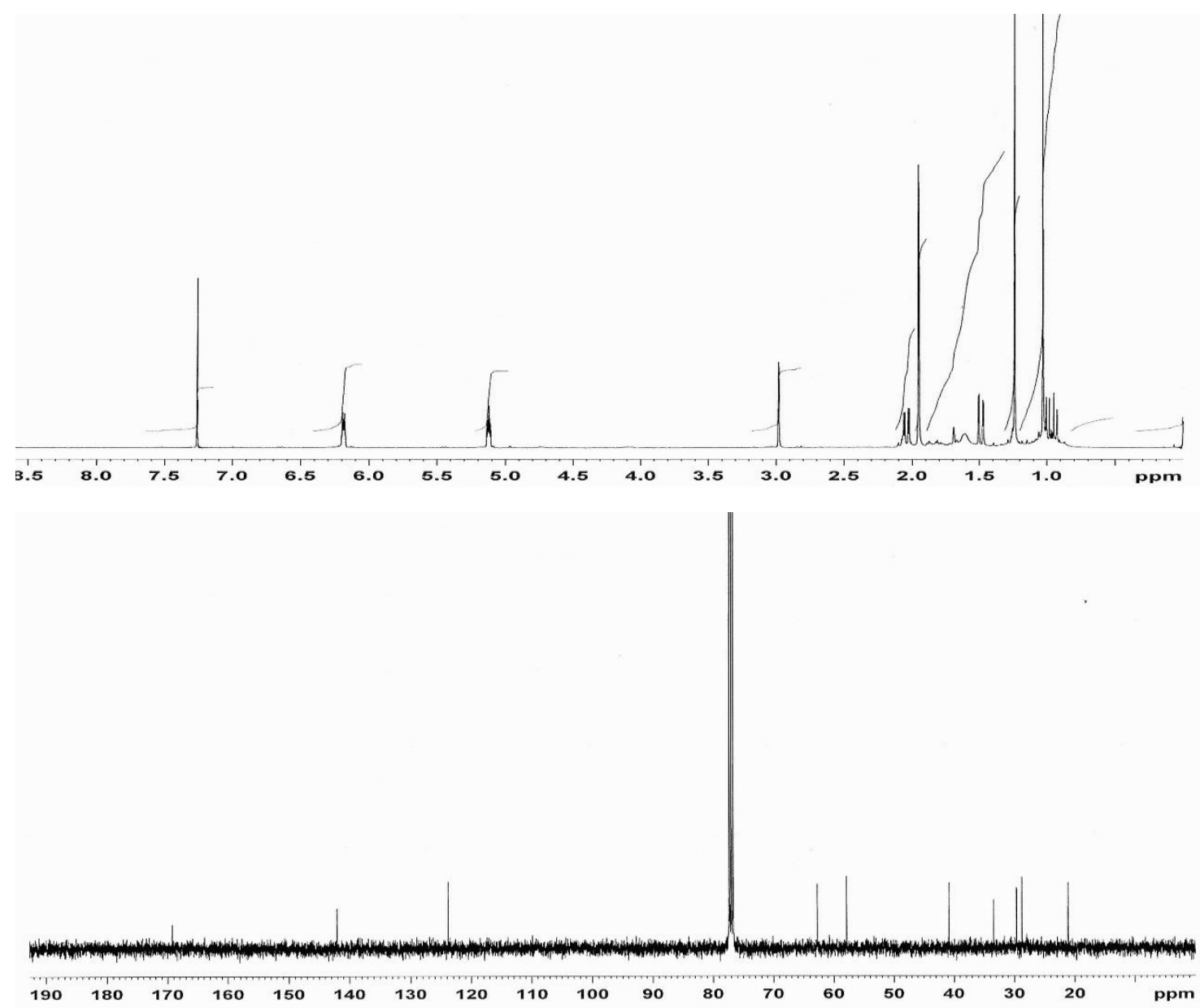

Şekil 6. 3,5,5-Trimetilsiklohek-2-en-1-il (azidosülfonil)karbamat (2e) bileşiğine ait ${ }^{1} \mathrm{H}-\mathrm{NMR}$ ve ${ }^{13} \mathrm{C}-\mathrm{NMR}$ Spektrumları 

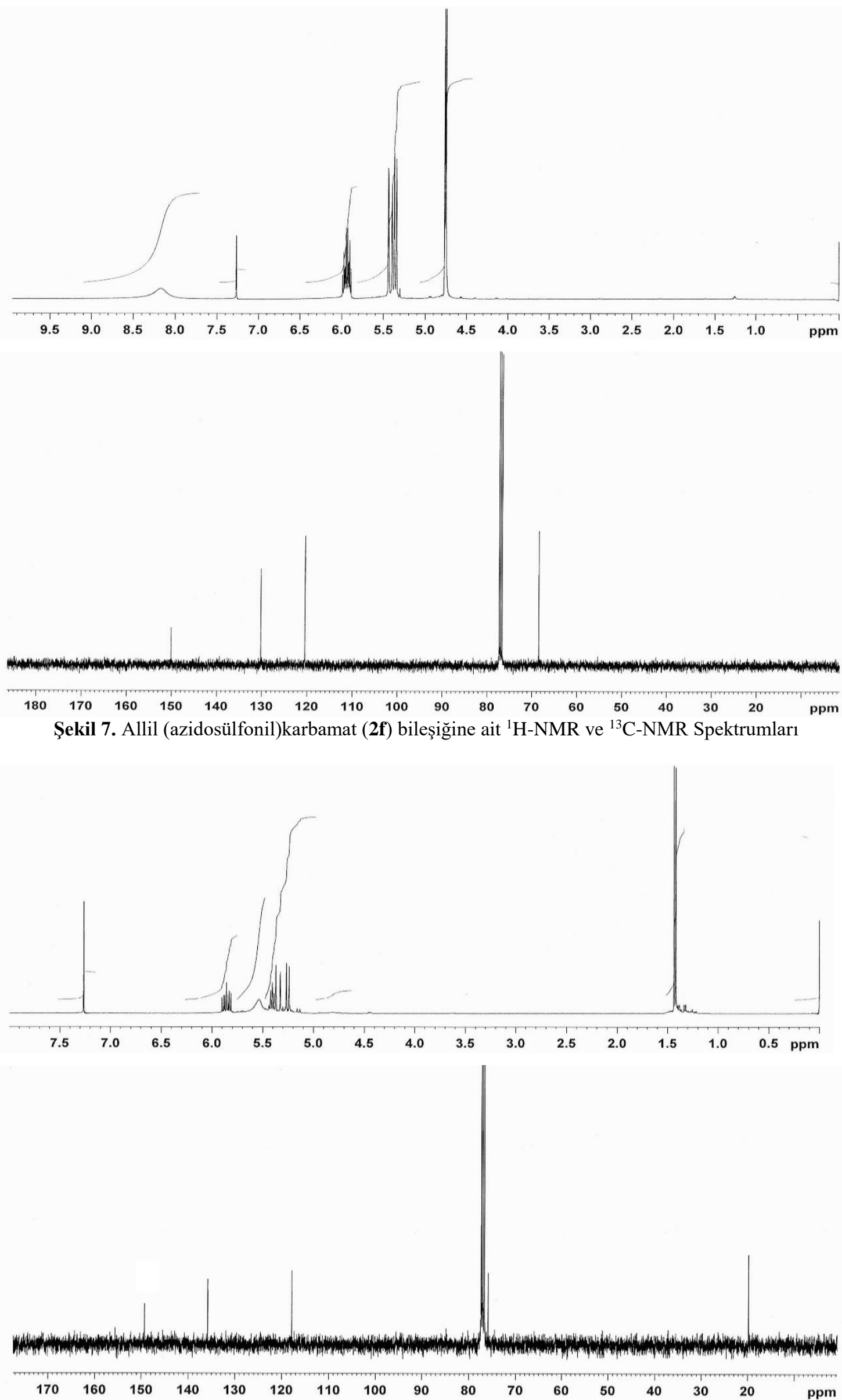

Şekil 8. Büt-3-en-2-il (azidosülfonil)karbamat (2g) bileşiğine ait ${ }^{1} \mathrm{H}-\mathrm{NMR}$ ve ${ }^{13} \mathrm{C}-\mathrm{NMR}$ Spektrumları 

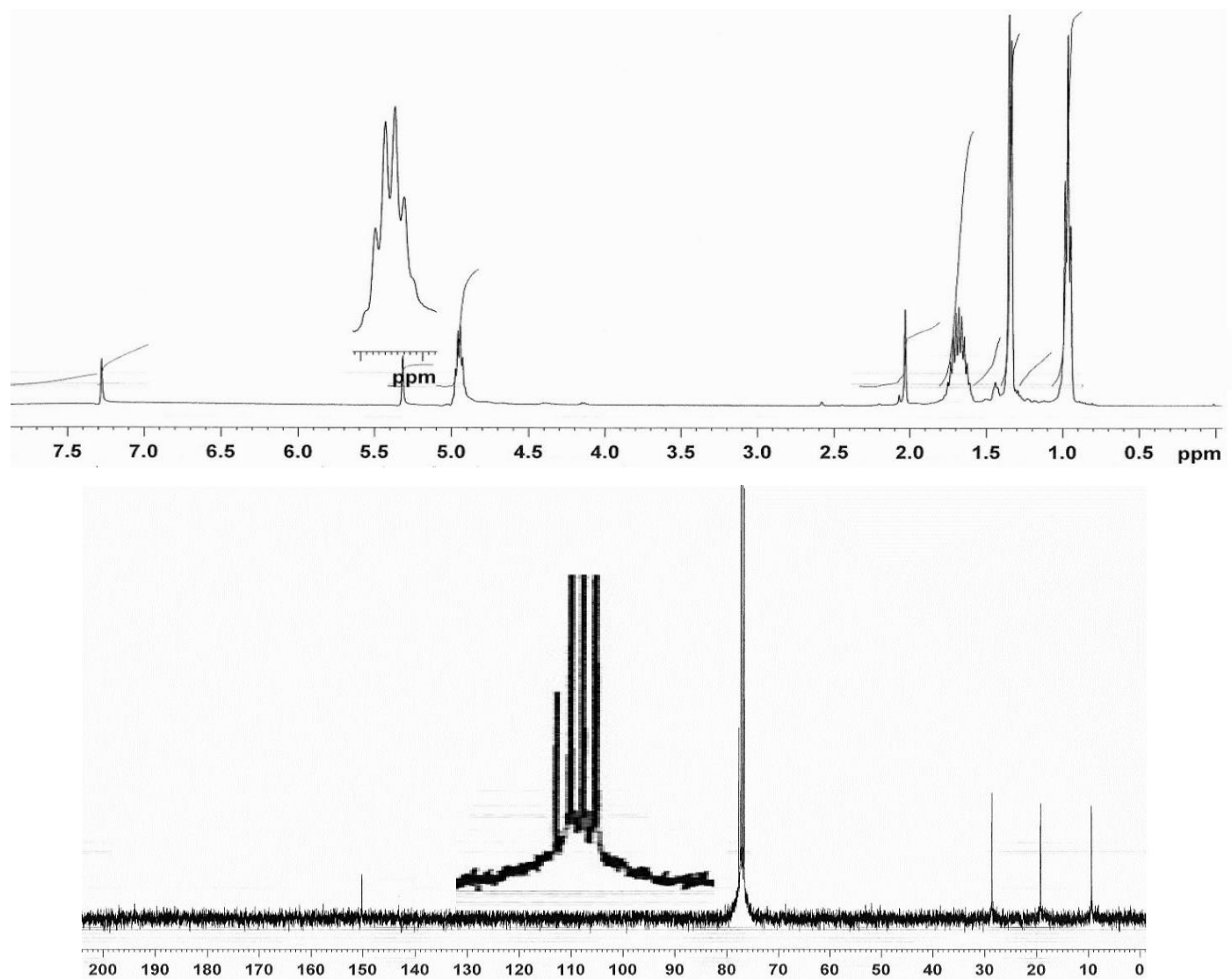

Şekil 9. 2-Bütil (azidosülfonil)karbamat (2h) bileşiğine ait ${ }^{1} \mathrm{H}-\mathrm{NMR}$ ve ${ }^{13} \mathrm{C}-\mathrm{NMR}$ Spektrumları

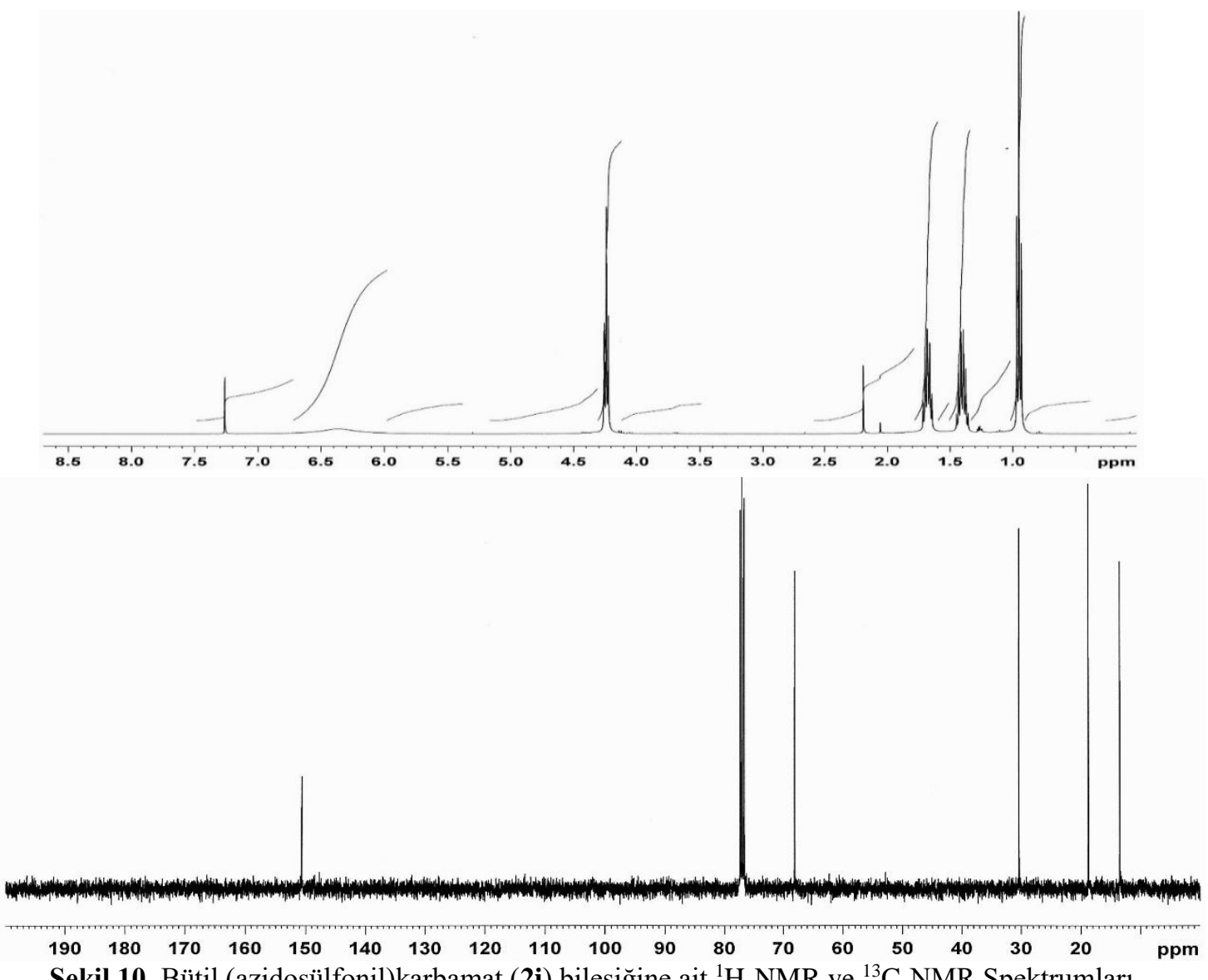




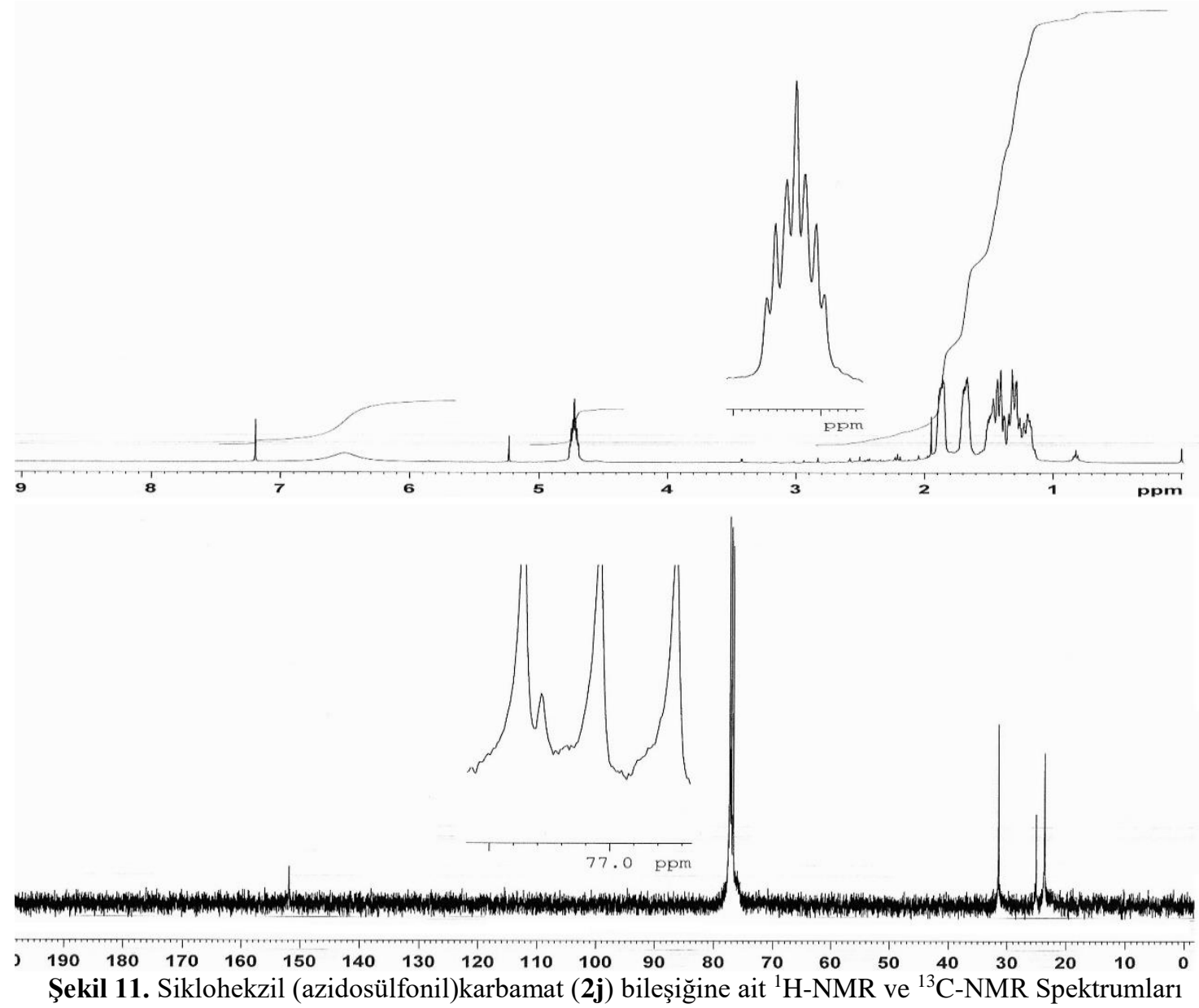

\section{BULGULAR VE TARTIŞMA}

Bu çalışmada, azidosülfonil bileşiklerin sentezi için çeşitli alkollerin asidik, bazik ve çözücü etkisi altında optimizasyon çalışmaları sonucunda, klorosülfonil izosiyanat ve sodyum azit varlığında, 1lıman şartlarda, yüksek verimle yeni azidosülfonil bileşiklerin sentezi incelenmiştir.

Optimizasyon çalışmaları için Benzil alkol (1a) ile Klorosülfonil izosiyanat'nın (CSI) reaksiyonu model olarak seçilmiş ve sonuçlar Tablo 1'de gösterilmiştir.

Tablo 1. Benzil(azidosülfonil)karbamat (2a) bileşiğinin sentezi için optimizasyon çalışması

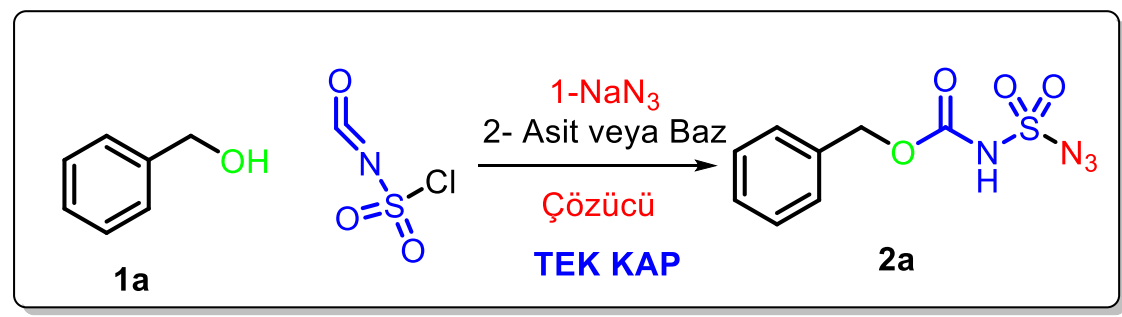

Optimizasyon için model reaksiyonda ilk olarak çözücü etkisi incelendi. Bunun için sırasıyla, diklorometan, asetonitril ve toluen çözücülerinde ve herhangi bir asit veya baz katalizörü kullanılmadan, oda sıcaklığında gerçekleştirilen reaksiyon sonucunda benzil (azidosülfonil)karbamat (2a) bileşiği sırasyla \%52 (Diklorometan), \%70 (Asetonitril) ve \%23 (Toluen) verimler ile sentezlenmiştir (Tablo 1, Giriş 1,7 ve 13). Bu model reaksiyonlar sonucunda en uygun çözücünün asetonitril olduğu belirlenmiştir. Fakat çözücü ve katalizörün etkisinin tek kapta reaksiyon verimlerinin değişebileceği 
literatürlerden bilindiği üzere her bir çözücü ve katalizör etkileri de ayrı ayrı incelenmiş ve Tablo 1'de özetlenmiştir.

\begin{tabular}{|c|c|c|c|}
\hline Giriş & Çözücü & Katalizör & Verim\% \\
\hline 1 & \multirow{6}{*}{$\mathrm{CH}_{2} \mathrm{Cl}_{2}$} & - & 52 \\
\hline 2 & & $\mathrm{AcOH}$ & 67 \\
\hline 3 & & TFA & 69 \\
\hline 4 & & $\mathrm{TfOH}$ & 79 \\
\hline 5 & & Piridin & 53 \\
\hline 6 & & $\mathrm{NEt}_{3}$ & 45 \\
\hline 7 & \multirow{6}{*}{$\mathrm{CH}_{3} \mathrm{CN}$} & - & 70 \\
\hline 8 & & $\mathrm{AcOH}$ & 74 \\
\hline 9 & & TFA & 83 \\
\hline 10 & & TfOH & 92 \\
\hline 11 & & Piridin & 43 \\
\hline 12 & & $\mathrm{NEt}_{3}$ & 35 \\
\hline 13 & \multirow{6}{*}{ Toluen } & - & 23 \\
\hline 14 & & $\mathrm{AcOH}$ & 46 \\
\hline 15 & & TFA & 49 \\
\hline 16 & & $\mathrm{TfOH}$ & 54 \\
\hline 17 & & Piridin & - \\
\hline 18 & & $\mathrm{NEt}_{3}$ & - \\
\hline
\end{tabular}

Diklorometan çözücüsü ve herhangi bir katalizör kullanılmadan, oda sıcaklığında gerçekleştirilen reaksiyon sonucunda benzil (azidosülfonil)karbamat (2a) bileşiği \%52 verim ile sentezlendiğini belirlemiştik. Sonrasında aynı çözücü ve sıcaklık şartlarında reaksiyon, sırasıyla Asetik Asit (AcOH), Trifloroasetik asit (TFA), Triflik asit (TfOH), Piridin ve Trietilamin ( $\mathrm{NEt}_{3}$ ) ile yapılmıştır (Tablo 1, Giriş 2-6). Bu reaksiyonlar sonucunda beklenen ürün oluşumu katalizörün kullanılmadığı reaksiyonlara göre, baz katalize reaksiyonlarda yaklaşık olarak yakın verimlerde, asit katalize reaksiyonlarda ise artığ gözlemlenmiştir. Fakat verimler istenilen değerlerde değildi.

Ardından ikinci çözücü olan asetonitril içerisinde yapılan reaksiyonlarda ise, oda sıcaklığında gerçekleştirilen reaksiyon sonucunda benzil (azidosülfonil)karbamat (2a) bileşiği \%70 verim ile sentezlendiğini belirlemiştik. Asetonitril çözücüsü içinde, sırasıyla Asetik Asit (AcOH), Trifloroasetik asit (TFA), Triflik asit (TfOH), Piridin ve Trietil amin $\left(\mathrm{NEt}_{3}\right)$ katalizörleri varlığında tepkimeler gerçekleştirildi (Tablo 1, Giriş 8-12). Bu reaksiyonlar sonucunda da beklenen ürününün oluşumu asit katalize reaksiyonlarda artığı, baz katalize reaksiyonlarda ise azaldığı gözlemlenmiştir. Ayrıca asetonitril içerisinde, katalizör olarak TfOH'ın kullanıldığı reaksiyonda \%92 verimle beklenen ürün çok iyi bir verimle elde edilmiştir (Tablo 1, Giriş 10). Bu verim literatür içinde çok iyi bir sonuç olduğu belirlenmiştir. Daha sonra Toluen çözücüsünde optimizasyon reaksiyonları (Tablo 1, Giriş 13-18) gerçekleştirilmiş, fakat elde edilen verimler diklorometan ve asetonitril çözücüsüne göre daha düşük olduğu gözlemlenmiştir. 
$\mathrm{Bu}$ elde edilen sonuçların 1şığında, çözücü olarak asetonitirilin, katalizör olarak da TfOH'ın kullanıldığı ve oda sıcaklığında, 8 saat süre ile gerçekleştirilen reaksiyonun en iyi yöntem olduğu belirlenmiştir (Tablo 1, Giriş 10).
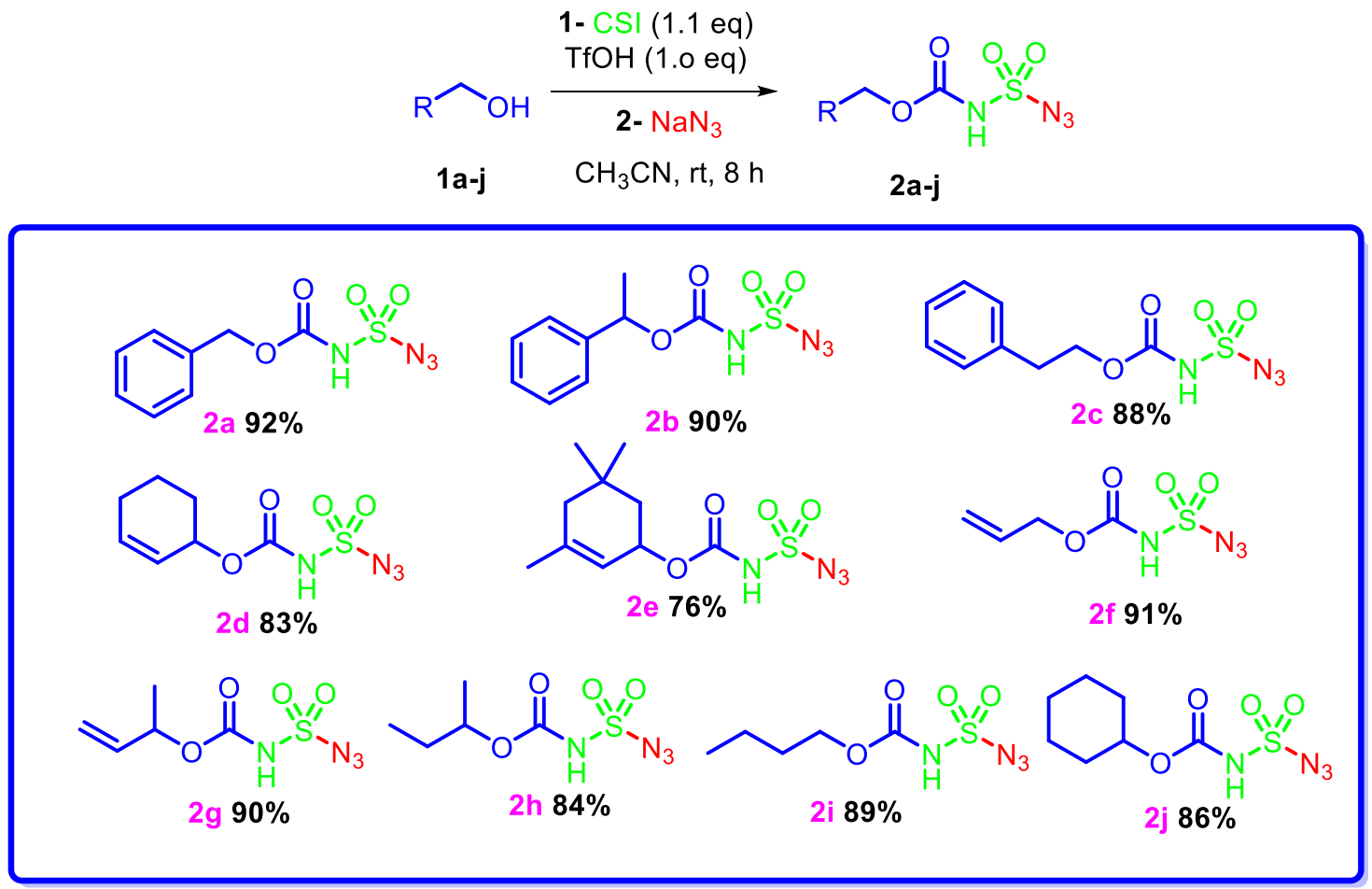

Şekil 12. Sentezi gerçekleştirilen azidosülfonil bileşikleri (2a-j)

Elde edilen optimize şartlarda çeşitli alkollerin (benzilik alkoller, alilik alkoller, sekonder ve primer alkoller) (1a-j) CSI ile olan tepkimeleri sonucu azidosülfonil bileşikleri (2a-j) yüksek verimlerle, tek kademede ve 1lıman şartlarda gerçekleştirildi (Şekil 12).

\section{SONUÇ}

Sonuç olarak, alkollerin CSI ve sodyum azit ile reaksiyonu sonucu, azidosülfonil bileşiklerinin sentezi için basit ve etkili bir yöntem geliştirildi. Bu yöntem için en uygun çözücünün asetonitril ve asidin $\mathrm{TfOH}$ olduğu belirlendi. Bu yöntem literatürde ilk olması ve reaksiyonun tek kap içerisinde gerçekleşmesi organik kimya ve ilaç sanayinde büyük önem arz etmektedir.

\section{TEŞEKKÜR}

Bu çalışma Atatürk Üniversitesi Bilimsel Araştırma Projesi (BAP-FBA-2018-6964) tarafindan desteklenmiş ve Atatürk Üniversitesi Fen Fakültesi Kimya Bölümünde gerçekleştirilmiştir.

\section{KAYNAKLAR}

Akbaba Y, Bastem E, Topal F, Gulcin I, Maras A, Goksu S, 2014. Synthesis and Carbonic Anhydrase Inhibitory Effects of Novel Sulfamides Derived from 1-Aminoindanes and Anilines. Archiv Der Pharmazie, 347: 95057.

Bauer JM, Frey W, Peters R, 2016. Dual Palladium(II)/Tertiary Amine Catalysis for Asymmetric Regioselective Rearrangements of Allylic Carbamates. Chemistry-a European Journal, 22: 5767-77.

Culhane JC, Fokin VV, 2011. Synthesis and Reactivity of Sulfamoyl Azides and 1-Sulfamoyl-1,2,3-triazoles. Organic Letters, 13: 4578-80. 
Daryadel S, Atmaca U, Taslimi P, Gulcin I, Celik M, 2018. Novel sulfamate derivatives of menthol: Synthesis, characterization, and cholinesterases and carbonic anhydrase enzymes inhibition properties. Archiv Der Pharmazie, 351.

Goddard-Borger ED, Stick RV, 2007. An efficient, inexpensive, and shelf-stable diazotransfer reagent: Imidazole1-sulfonyl azide hydrochloride. Organic Letters, 9: 3797-800.

Golob T, Liebl R, von Angerer E, 2002. Sulfamoyloxy-substituted 2-phenylindoles: Antiestrogen-based inhibitors of the steroid sulfatase in human breast cancer cells. Bioorganic \& Medicinal Chemistry, 10: 3941-53.

Hartwig JF 1998. Carbon-heteroatom bond-forming reductive eliminations of amines, ethers, and sulfides. Accounts of Chemical Research, 31: 852-60.

Kienle M, Dubbaka SR, Brade K, Knochel P, 2007. Modern amination reactions. European Journal of Organic Chemistry: 4166-76.

Matier WL, Comer WT, Deitchman D, 1972. Sulfamoyl Azides-Hydrolysis Rates and Hypotensive Activity. Journal of Medicinal Chemistry, 15: 538.

Wolf OT, Kirschbaum C, 1999. Actions of del dehydroepiandrosterone and its sulfate in the central nervous system: effects on cognition and emotion in animals and humans. Brain Research Reviews, 30: 264-88.

Zhao J, Li ZY, Song SL, Wang MA, Fu B, Zhang ZH, 2016. Product-Derived Bimetallic Palladium Complex Catalyzes Direct Carbonylation of Sulfonylazides. Angewandte Chemie-International Edition, 55: 5545-49. 